\title{
Antecedentes da compra compulsiva dos universitários em Mariana (MG)
}

\author{
Antecedents of compulsive buying of universities in Mariana (MG)
}

\author{
Israel José dos Santos Felipe ${ }^{1}$ \\ Harrison Bachion Ceribeli ${ }^{2}$ \\ Rafaela Maciel Brandão ${ }^{3}$
}

\begin{abstract}
Resumo
No Brasil, o número de famílias endividadas aumentou substancialmente nos últimos anos. Isso pode ser explicado, dentre outros fatores, pelo aumento do comportamento de compra compulsiva na sociedade atual. Nesse contexto, o objetivo desta pesquisa foi analisar os antecedentes do comportamento compulsivo de compra de jovens universitários de uma Instituição Federal de Ensino localizada em Mariana (MG). Dentre os vários fatores que levam os indivíduos a comprarem compulsivamente, foram utilizados três como base para este estudo: busca por poder e prestígio, ansiedade e desconfiança. Como método de coleta de dados, realizou-se um levantamento de campo. Para análise de dados, optou-se pela modelagem de equações estruturais, por meio da aplicação de duas abordagens: análise fatorial confirmatória e análise de caminhos. $A$ partir do referencial teórico construído e da análise dos resultados obtidos, foi possível confirmar a ansiedade e a desconfiança como antecedentes do comportamento de compra compulsivo entre os jovens universitários; o primeiro exercendo influência positiva, e o segundo, negativa.
\end{abstract}

Palavras-chave: Comportamento do consumidor universitário.Compra compulsiva.Consumo compulsivo.

\begin{abstract}
In Brazil, the number of indebted households has increased substantially in recent years, which may be explained, among other factors, by the increase in compulsive buying behavior in society today. In this context, the objective of this research was to analyze the antecedents of compulsive buying behavior of university students of an Education Federal Institution located in Mariana (MG).Among the various factors that lead individuals to buy compulsively, we use three as the basis for this study: search for power and prestige, anxiety and mistrust.As data collection method, we chose to conduct a field survey. For data analysis, we opted for Structural Equation Modeling, through the application of two approaches: Confirmatory Factor Analysis and Path Analysis. From the theoretical framework and analysis of the results, it was possible to confirm the anxiety and distrust as antecedents of compulsive buying behavior among university students, the first exerting positive influence and the second negative.
\end{abstract}

Keywords: Behavior of university consumer. Compulsive shopping. Compulsive consumption.

\section{Introdução}

O interesse por estudos relacionados ao comportamento do consumidor vem aumentando consideravelmente ao longo dos anos, devido ao fato de o consumo ter se tornado, atualmente, algo inerente à vida dos seres humanos. Estudiosos de várias áreas do conhecimento, como Administração, Economia e Psicologia, tentam explicar o comportamento do consumidor por meio de teorias diversas,

Doutorando em Administração pela Fundação Getúlio Vargas (FGV-EAESP) Mestre em Administração pela Universidade Federal do Rio Grande do Norte (UFRN) Professor Assistente da Universidade Federal de Ouro Preto (UFOP). Brasil. Afiliação: Universidade Federal de Ouro Preto. Lattes: http://lattes.cnpq.br/4608734331831234 Email: israeljfelipe@gmail.com

2 Doutor em Administração pela Universidade de São Paulo (FEARP-USP) Professor Adjunto da Universidade Federal de Ouro Preto (UFOP) Vice-diretor do Instituto de Ciências Sociais Aplicadas (ICSA-UFOP). Brasil. Afiliação: Universidade Federal de Ouro Preto. Lattes: http://lattes. cnpq.br/8913367429929208 Email: harrisonbceribeli@hotmail.com

3 Graduada em Administração pela Universidade Federal de Ouro Preto (UFOP). Brasil. Afiliação: Universidade Federal de Ouro Preto. Lattes: http://lattes.cnpq.br/5858508505794874 Email: rafaelamabrandao@hotmail.com 
o que deu origem a diferentes vertentes de investigação, ancoradas em aspectos econômicos, sociais e psicológicos, que podem ser complementadas pelos aspectos pessoais e culturais, conforme argumentam De Medeiros et al. (2015), Kunkel et al. (2015), Vieira et al. (2015), Merlo e Ceribeli(2014), Baker et al. (2013), Gudmunson e Beutler (2012), Kukar-Kinney, Ridgway e Monroe (2012), Leite et al. (2012), Roberts e Roberts (2012), Hanna, Wozniack e Hanna (2009), Solomon (2008), Blackwell, Miniard e Engel (2005), Mowen e Minor (2003) e Sheth, Mittal (ANO?).

De acordo com Stefanini e Oliveira (2014), dentre os temas relacionados ao comportamento do consumidor, a compra compulsiva é um dos mais antigos e o mais estudado. Medeiros et al. (2015) afirmam que é grande o número de pesquisas acerca do tema compra compulsiva, que pode ser conceituada como aquela em que se adquire algo do qual não se tem necessidade e pela qual não se pode pagar (MITTAL et al., 2008). Segundo Verplanken et al. (2012), a compra compulsiva é um processo por meio do qual o consumidor busca a compra para se sentir melhor e obter sensação de prazer momentâneo.

Por meio da compra compulsiva, o consumidor tenta suprir sentimentos negativos ou lacunas emocionais, buscando a sensação de satisfação e prazer associada ao consumo; motivo pelo qual compradores compulsivos geralmente são ansiosos e consomem para aliviar tensões, ansiedade ou desconforto (MEDEIROS et al., 2015). Cabe acrescentar que as compras compulsivas resultam de impulsos não controlados, distúrbios, ou transtornos dos indivíduos (BAKER et al., 2013).

Constantemente, os indivíduos tentam solucionar seus problemas através do consumo, devido aos contínuos estímulos veiculados pelos meios de comunicação modernos, provocando altos níveis de atitudes consumistas entre os jovens e adolescentes (BEUTLER; GUDMUNSON, 2012). É por isso que a educação financeira das crianças e jovens vem se tornando tema cada vez mais comum quando se analisa o que vem acontecendo na sociedade moderna. A educação financeira pode ajudar a reduzir um grande problema dos brasileiros, que é o endividamento elevado. Esse problema, que é originado, dentre outros fatores, pelo materialismo, pelas compras compulsivas, pelo fácil acesso ao crédito e ao cartão de crédito e pelos baixos níveis de alfabetização financeira, apresenta como consequência direta no baixo nível de bem-estar financeiro e no surgimento de muitas emoções negativas (KUNKEL; VIEIRA; POTRICH, 2015).

De acordo com a Pesquisa de Endividamento e Inadimplência do Consumidor (Peic), da Associação Brasileira das Empresas de Cartão de Crédito e Serviços (ABECS, 2015), o número de famílias brasileiras endividadas alcançou $62,7 \%$ em 2015 . Se, por um lado, a população adulta é a grande protagonista dessa história e precisa ser educada financeiramente; por outro, as crianças e jovens devem também ser o foco da educação financeira, visando à formação de uma sociedade mais consciente e sustentável em um futuro próximo, revertendo-se o quadro atual, que, de acordo com Roberts e Jones (2001), caracteriza-se pelo aumento dos problemas financeiros pessoais entre os indivíduos com menos de trinta e cinco anos de idade, corroborando a afirmação de Stefanini e Oliveira (2014) de que indivíduos mais jovens têm maior tendência ao comportamento de compra compulsiva.

Nesse contexto de endividamento observado principalmente entre os mais jovens, buscou-se responder à seguinte questão de pesquisa: Quais são os antecedentes do comportamento de compra compulsiva entre os jovens universitários? A partir dessa problemática, delimitou-se como objetivo desta pesquisa analisar os antecedentes do comportamento compulsivo de compra de jovens universitários de uma Instituição Federal de Ensino localizada em Mariana (MG). Dentre os vários fatores que levam os indivíduos a comprarem compulsivamente, foram utilizados três como base para o estudo: busca por poder e prestígio, ansiedade e desconfiança.

$\mathrm{Na}$ mesma linha de investigação de Roberts e Jones (2001), analisou-se estatisticamente a influência dessas três dimensões, que fazem parte da Escala de Atitudes Financeiras (Money Attitude Scale) desenvolvida por Yamauchi e Templer (1982), no comportamento de compra compulsiva dos jovens universitários.

Como justificativa para esse trabalho destaca-se que, apesar da existência de vários estudos relacionados ao comportamento do consumidor jovem, ainda não se conseguiu compreender com exatidão as causas e antecedentes do comportamento de compra compulsiva desse público. Além disso, o tema apresenta diversos aspectos que merecem melhor esclarecimento e profundo mergulho 
investigativo (KUKAR-KINNER; RIDGWAY; MONROET, 2012), sendo que, por meio de pesquisas focadas na compreensão desse comportamento, é possível tentar evitá-lo, pois, ao conhecer seus antecedentes, é possível inibi-los, o que também justifica a motivação apara a realização deste estudo.

A relevância deste estudo reside principalmente na possibilidade de levantar os fatores que motivam o comportamento de compra compulsiva, para que seja possível repensar os valores atuais que têm levado os indivíduos a se endividarem cada vez mais cedo. Esse, sem dúvida, é o primeiro passo para se restabelecer o equilíbrio financeiro na vida dos brasileiros.

\section{Revisão da literatura}

O consumo tem se tornado cada vez mais intenso, sendo um reflexo de fatores culturais, sociais e individuais. De acordo com Gudmunson e Beutler (2012), Manolis e Roberts (2012) e Roberts e Jones (2001), o consumo está associado a fatores da conjuntura social atual, como o acesso ao crédito, e a aspectos relacionados aos desejos dos indivíduos. Os mesmos autores reforçam essa ideia, afirmando que a aquisição de bens não tem função apenas de satisfação de necessidades, mas representa também a busca pela identidade do indivíduo. Com isso, evidencia-se a necessidade de se avançar nas pesquisas acerca do comportamento do consumidor na sociedade atual.

Segundo Blackwell, Miniard e Engel (2005), a área de estudos do comportamento do consumidor tem como escopo as atividades desempenhadas para se obter, consumir e dispor de produtos e serviços, o que engloba os processos de decisão que antecedem e sucedem estas ações. Portanto, todo o processo decisório envolvido antes do consumo até as reações geradas no pós-compra são temáticas interessantes ao estudo do comportamento do consumidor.

Diversos são os fatores que exercem influência sobre os consumidores na atualidade (MERLO; CERIBELI, 2014). Fatores econômicos, como emprego, salário e disponibilidade de crédito, têm implicação direta no comportamento do consumidor, aumentando, ou diminuindo, o poder de compra das famílias, influenciando a disposição para comprar e levando à antecipação, ou adiamento, do consumo. Somam-se a tais fatores o custo do dinheiro e consequentemente do consumo, que sofre influência da inflação, das taxas de juros e das taxas de câmbio (HANNA; WOZNIACK; HANNA, 2009).

Outros fatores mercadológicos que influenciam o comportamento do consumidor são as políticas monetária e fiscal, as regulamentações econômicas e ambientais, e a tecnologia, que permite que sejam explorados nichos de mercado cada vez mais variados a fim de atender diferentes demandas (SOLOMON, 2008).

O fator cultural também exerce papel importante no comportamento de consumo, pois os valores de cada indivíduo, ou sociedade, impactam a forma como consomem e suas motivações para tal (HANNA; WOZNIACK; HANNA, 2009; MERLO; CERIBELI, 2014). Conforme Medeiros et al. (2015), em um contexto cultural que se caracteriza por valores materialistas, o consumo é altamente estimulado.

De forma complementar, os fatores sociais também influenciam os consumidores, não só durante o processo decisório de compra, como também em suas atitudes e comportamentos. Segundo Sheth, Mittal e Newman (2001) e Solomon (2008), os indivíduos são profundamente influenciados por diferentes grupos, seja se expondo a novos comportamentos e estilos de vida, alterando sua autoimagem, seja tentando se enquadrar a grupos aos quais ainda não pertence.

Os fatores pessoais, como idade, gênero, profissão, personalidade, estilo de vida, entre outros, podem ser agrupados em outro conjunto de elementos que influenciam as decisões de consumo (MOWEN; MINOR, 2003). Solomon (2008) acredita que, ao adquirir bens, o consumidor busca consolidar sua identidade e projetá-la perante a sociedade.

Por fim, têm-se os fatores psicológicas que, de acordo com Blackwell, Miniard e Engel (2005), estão ligados às motivações do indivíduo, sua percepção diante das situações e dos objetos de consumo, sua aprendizagem e memória de experiências anteriores. Para Medeiros et al. (2015), a compra motivada por fatores psicológicos é característica do consumidor que se utiliza do consumo para melhorar a autoestima, autoimagem e seus relacionamentos. 
Especificamente no caso das compras compulsivas, são muitos os elementos que podem ser apontados como antecedentes, visto que elas podem estar ligadas à emoções e sentimentos, estímulos no local da compra, moda, materialismo, cultura, idade, entre outros (STEFANINI; OLIVEIRA, 2014).

Para Roberts (1998), os fatores que contribuem para a ocorrência do consumo compulsivo podem ser agrupados em três categorias: a primeira se refere às influências psicológicas, incluindo autoestima, percepção do status social e fantasias; a segunda está relacionada às influências familiares; por fim, a terceira diz respeito às influências sociológicas, envolvendo a pressão dos pares, as mídias e o uso e do cartão de crédito.

Rook e Fischer (1995) afirmam que o comportamento de efetuar uma compra sem planejamento e sem necessidade é um traço de personalidade frequentemente presente nos indivíduos que vivem nas sociedades contemporâneas, podendo ser considerado como uma compulsão. Por sua vez, Roberts e Jones (2001) afirmam que uma comunidade de devedores cria um ambiente que reforça as crenças, atitudes e normas de comportamento que justificam os gastos excessivos, como o excesso de compras, que passam a ser aceitáveis.

De acordo com a pesquisa de Elliott (1994), a tendência ao vício do consumo guarda relação com inseguranças, problemas de infância, o fato de a pessoa se sentir desinteressante, depressão e falta de apoio emocional nos relacionamentos. Segundo Roberts e Jones (2001), tem consequências potencialmente graves para o indivíduo afetado, o que vem ao encontro dos argumentos de Dittmar, Long e Bond (2007), que indicam que os compradores compulsivos não têm controle sobre sua vontade de comprar, mesmo estando conscientes das consequências negativas que podem incidir sobre sua vida financeira, social e pessoal caso ajam imprudentemente.

Valence, D'Astous e Fortier (1998) verificaram que o sentimento de ansiedade é fator crítico no surgimento dessa conduta, haja vista que ele provoca ações espontâneas, empurrando o consumidor para a tomada de decisões que minimizem a tensão e os sentimentos negativos. De maneira complementar, Oliveira, Ikeda e Santos (2004) constataram que jovens com necessidade de se autoafirmarem e mostrarem prestígio usam o dinheiro para influenciarem e impressionarem os outros.

Com o intuito de investigarem os antecedentes da compra compulsiva entre jovens universitários, Roberts e Jones (2001) testaram três dimensões da escala Money Attitude Scale (MAS), criada por Yamauchi e Templer (1982): poder-prestígio, desconfiança e ansiedade.

No que se refere à dimensão poder-prestígio, diversos autores abordam a relação entre dinheiro, poder e status, sinalizando um excesso de importância conferida aos valores materiais. Conforme defendem Roberts e Jones (2001), para muitas pessoas, o dinheiro significa poder; onde o dinheiro está envolvido, seu valor principal é a sua utilidade na remoção de obstáculos que estejam no caminho da pessoa em busca do poder. Seguindo a mesma linha, Oliveira, Ikeda e Santos (2004) afirmam que, para muitos jovens, o dinheiro é sinônimo de status e poder, e uma ferramenta para obter respeito em seus grupos de convivência. Esses mesmos autores complementam que, para alguns indivíduos, o ato da compra é obviamente um símbolo de status, resultante de sua habilidade de comprar produtos sofisticados.

A busca pelo poder e prestígio acaba se tornando uma maratona sem fim, tendo em vista que a produção de bens de consumo aumenta e se renova constantemente, fazendo com que o indivíduo tenha que adquirir cada vez mais produtos para impressionar aos outros. Roberts e Jones (2001) afirmam que, enquanto os outros também estão tentando sinalizar o seu poder social, possuindo e exibindo bens materiais, o nível de bens necessários para fazer uma poderosa afirmação social aumenta continuamente.

Por sua vez, a dimensão desconfiança refere-se ao grau de suspeita que o indivíduo tem em relação ao preço de um produto e as vantagens em adquiri-lo, ou seja, ao contrário do comprador compulsivo, as pessoas que se encaixam nessa dimensão levam em consideração o preço do produto na hora da compra, o seu custo-benefício e se sua aquisição é realmente necessária (YAMAUCHI; TEMPLER, 1982).

De acordo com Weinberg e Gottwald (1982), os compradores compulsivos demonstram mais emoção, entusiasmo, alegria e prazer ao comprar quando comparados aos compradores planejados. Essa ideia é reforçada por Stefanini e Oliveira (2014), Wagner et al. (2013) e Verplanken e Sato (2011), os quais 
afirmam que os fatores que mais impulsionam os consumidores a comprarem compulsivamente são a emoção e o sentimento que proporciona, os quais não atingem compradores que planejam suas compras e que tendem a ser mais desconfiados.

Yamauchi e Templer (1982) descrevem que pessoas com alta pontuação nesse fator como hesitantes, suspeitos e duvidosos sobre situações que envolvem dinheiro. Os autores afirmam ainda que altas pontuações nessa dimensão também refletem uma falta de fé na própria capacidade para tomar decisões de compra eficientes.

Por fim, a dimensão ansiedade refere-se a um distúrbio psicológico motivador das compras compulsivas, pois os indivíduos buscam suprir suas carências adquirindo bens materiais dos quais não necessitam. Por essa razão, Verplankenet al. (2005) sugerem que o sentimento negativo também impulsiona o comprador, que se sente melhor após o consumo, por isso os compulsivos geralmente são excessivos e compram para aliviar tensão, ansiedade ou desconforto.

Segundo Byrne et al. (2007), Hutchinson, Baldwin e Oh (2006) e Edwards (1993), o comprador compulsivo apresenta um nível mais elevado de ansiedade em relação ao não compulsivo e realiza compras principalmente em períodos de maior estresse. Reforçando essa ideia, Roberts e Jones (2001) e Roberts e Roberts (2012) afirmam que compras compulsivas são vistas como uma solução rápida para a ansiedade, sendo o escape dessa sensação a principal motivação das pessoas que apresentam comportamentos viciosos ou compulsivos.

\section{Aspectos metodológicos}

Esta pesquisa pode ser classificada como descritiva de caráter quantitativo, por envolver técnica de coleta de dados objetivando descrever características de certa população e análise estatística dos dados. Para Hair et al. (2005), estudos de caráter descritivo são estruturados para medirem características descritas em uma questão de pesquisa, sendo que as teorias levantadas servem para conduzir o pesquisador acerca do que deve ser mensurado.

Malhotra (2006) afirma que as pesquisas descritivas são divididas basicamente em estudos transversais, pois envolvem a coleta de informações a partir de cada elemento que faz parte da amostra de pesquisa por uma única vez, e longitudinais, pois acessam os elementos fixos que fazem parte da amostra da pesquisa repetidas vezes, estudando as mesmas pessoas ao longo do tempo. Nesta pesquisa, optou-se pelo estudo transversal, no qual cada indivíduo será acessado uma única vez.

Como método de coleta de dados, realizou-se um levantamento de campo. A aplicação dos questionários foi realizada de forma presencial pelos pesquisadores, por meio da distribuição aos indivíduos selecionados para compor a amostra do presente estudo, selecionada de forma não probabilística, por conveniência.

As questões presentes no questionário foram elaboradas com base nos construtos teóricos identificados durante a revisão bibliográfica realizada nesta pesquisa. Na primeira parte do questionário, buscou-se conhecer o perfil do respondente com perguntas referentes à faixa etária, gênero e renda mensal; na segunda parte, foram inseridas questões adaptadas do estudo de Yamauchi e Templer (1982), mas cada um dos construtos analisados (poder-prestígio, desconfiança, ansiedade e compra compulsiva) foi desmembrado em quatro, cinco ou seis questões, dando origem a 20 variáveis de pesquisa.

A população-alvo escolhida para esta pesquisa foi o conjunto de estudantes universitários de uma universidade federal localizada em Mariana, cidade do estado de Minas Gerais. Tal escolha justifica-se pelo fato de ser uma parcela da sociedade que movimenta a economia local, considerando que, no caso de Mariana, a economia do município depende de forma considerável dos universitários. Cabe acrescentar que a ideia de selecionar esse público é reforçada também pelo estudo de Pallan et al. (2011), o qual relata que o público universitário apresenta uma gama de informações que ainda precisam ser exploradas no meio acadêmico.

Os universitários delimitados como população-alvo do estudo geram renda através do consumo de bens duráveis, bens não duráveis e serviços. Além disso, são jovens, em sua maioria, que convivem com 
o cartão de crédito desde muito novos, sendo uma ferramenta comum e presente no seu dia a dia, o que estimula o comportamento de compra compulsivo (PALLAN et al, 2011; ROBERTS; ROBERTS, 2012).

O tamanho da amostra foi definido considerando-se as recomendações de Hair Junior et al. (2009) de que deve haver pelo menos 10 elementos para cada variável de pesquisa. Considerando que há 20 variáveis de pesquisa, delimitou-se como 200 observações o tamanho mínimo da amostra.

Além da importância atribuída pelos jovens ao consumo como símbolo de poder e prestígio, levouse em consideração também outro fator que pode gerar um comportamento compulsivo de comprar e que também faz parte do dia a dia dos jovens universitários: a ansiedade. Por fim, agregou-se à pesquisa um fator que poderia afetar de forma inversa a compra compulsiva: a desconfiança que apresentam em relação a preços e custo-benefício dos produtos. Todos esses construtos foram reunidos em um modelo teórico, testado anteriormente por Roberts e Jones (2001), que pode ser observado na figura 1.

Figura 1- Atitudes relacionadas ao dinheiro e compras compulsivas

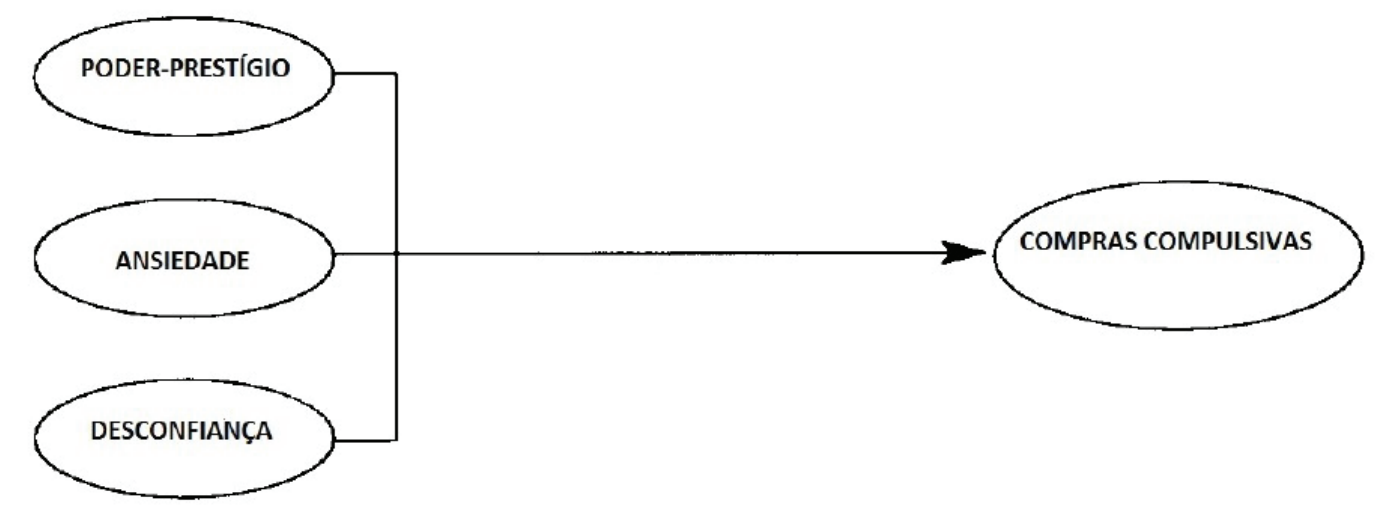

Fonte: Adaptado de Roberts e Jones (2001).

Para análise de dados, optou-se pela modelagem de equações estruturais (structural equation modeling), por meio da qual as variáveis observadas relacionadas aos construtos poder-prestígio, ansiedade e desconfiança foram agrupadas em variáveis latentes exógenas, as quais foram testadas como antecedentes da variável latente endógena compras compulsivas, sendo a última resultante do agrupamento das variáveis observadas relacionadas ao constructo.

\section{Apresentação e discussão dos resultados}

\subsection{Caracterização e análise da amostra}

A amostra de pesquisa foi composta por 218 estudantes universitários de determinado campus de uma universidade federal, localizado em Mariana (MG), que possui os seguintes cursos de graduação: Administração, Jornalismo, Economia e Serviço Social. A caracterização da amostra foi efetuada por meio do software IBM SPSS. Para analisar o perfil socioeconômico e demográfico, foram utilizadas variáveis como gênero, faixa etária, renda mensal, curso de graduação que está cursando e gasto médio mensal com cartão de crédito.

Com relação ao gênero dos respondentes, 137 são mulheres, o que equivale a aproximadamente $63 \%$ do total da amostra, e 81 são homens, aproximadamente $37 \%$ do total. Ao efetuar a análise cruzada das variáveis gênero e faixa etária, obteve-se o quadro 1 , que possibilita concluir que $58,7 \%$ dos respondentes estão na faixa etária de 21 a 25 anos, sendo que a maioria são mulheres. 
Quadro 1- Análise cruzada de gênero e faixa etária

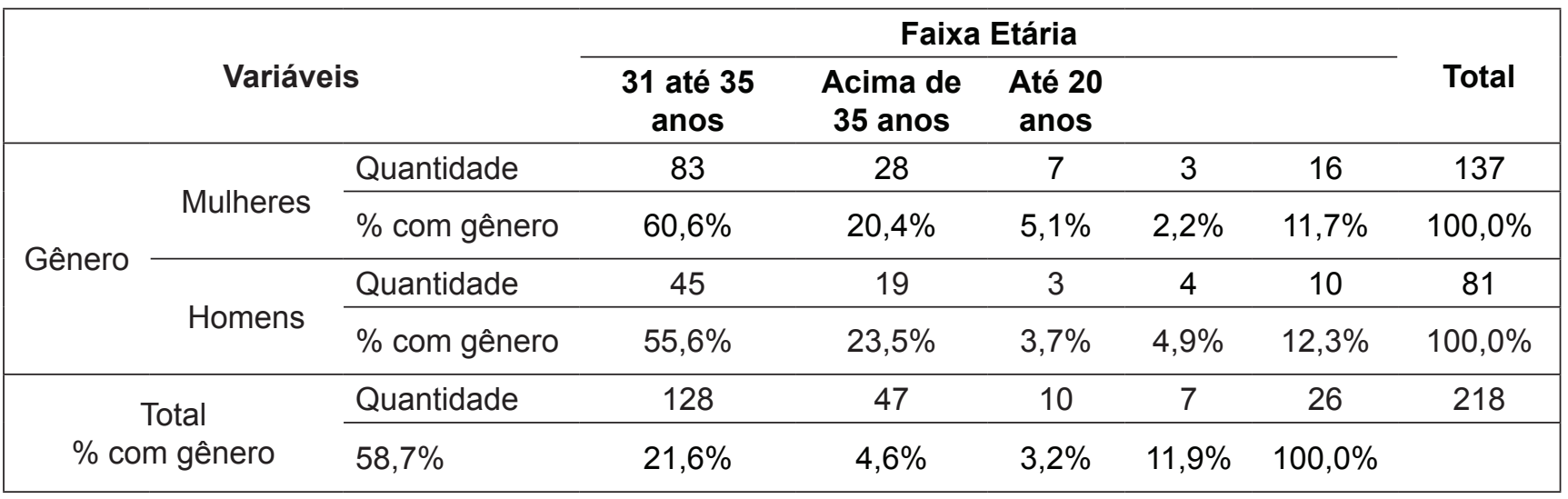

Fonte: Elaborado pelos autores (2016).

Aproximadamente $23 \%$ dos respondentes afirmaram não possuir renda própria e $61 \%$ possuem renda de até $\mathrm{R} \$ 2.000,00$ mensais. $90 \%$ da amostra têm um gasto médio com fatura de cartões de até $\mathrm{R} \$ 1.000,00$, sendo que, dentre eles, $24 \%$ não possuem renda própria.

Verificou-se, ao analisar de forma cruzada as variáveis renda mensal e curso, que a amostra é composta, em sua maioria, por estudantes do curso de Administração (45,9\%) e que, dentre os respondentes, são os que possuem maior renda.

Tratando-se da relação entre as compras e o estado emocional dos indivíduos, ao serem questionados se já haviam comprado algo para se sentirem melhor, $55,7 \%$ dos respondentes afirmaram que sim e, dentre eles, $62 \%$ são mulheres, conforme pode ser visualizado no gráfico 1 , o que leva à hipótese de que sentimentos negativos estimulam, principalmente, as mulheres a efetuarem compras.

Gráfico 1 - Gênero e compra compulsiva

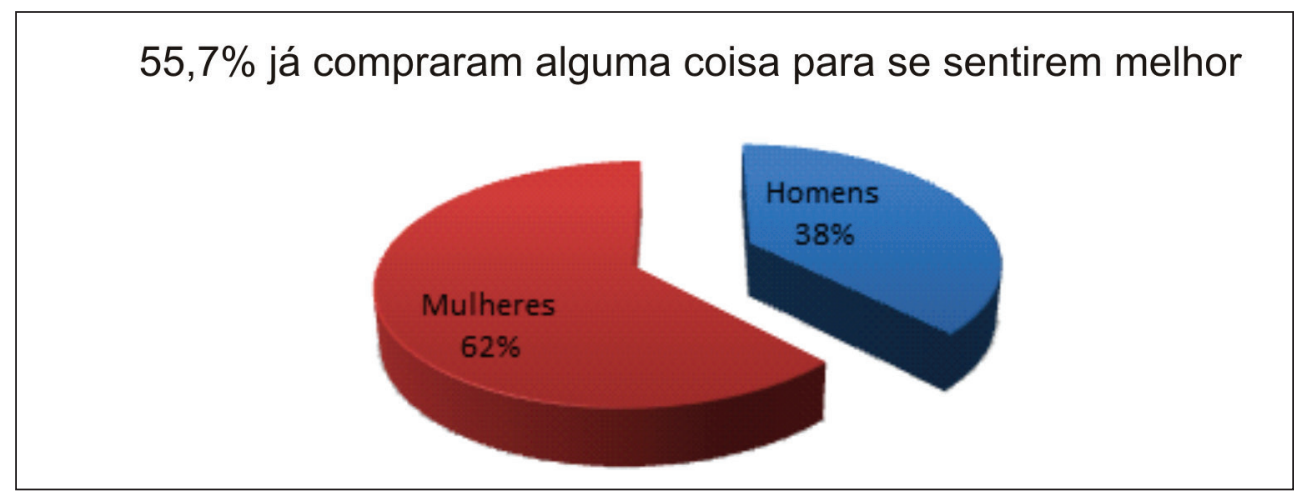

Fonte: Elaborado pelos autores (2016).

Com o intuito de verificar se os universitários que trabalham são menos compulsivos dos que os que não trabalham, cruzou-se a variável renda com uma das variáveis mais expressivas relacionadas à compra compulsiva, como pode ser observado no quadro 2. A partir dos resultados obtidos, depreendeuse que a variável renda não influencia o comportamento de compra compulsiva, pois não foi verificada nenhuma tendência que comprove essa hipótese, embora sejam necessários estudos mais aprofundados que possam embasar melhor essa conclusão. Apesar disso, foi possível identificar que indivíduos com renda de até $R \$ 2.000,00$ são os que mais tendem a efetuar compras compulsivas, sendo eles a maioria dos que concordaram (4) ou concordaram totalmente (5) com a afirmativa "Já comprei alguma coisa para me sentir melhor". 
Quadro 2 - Renda e compra compulsiva

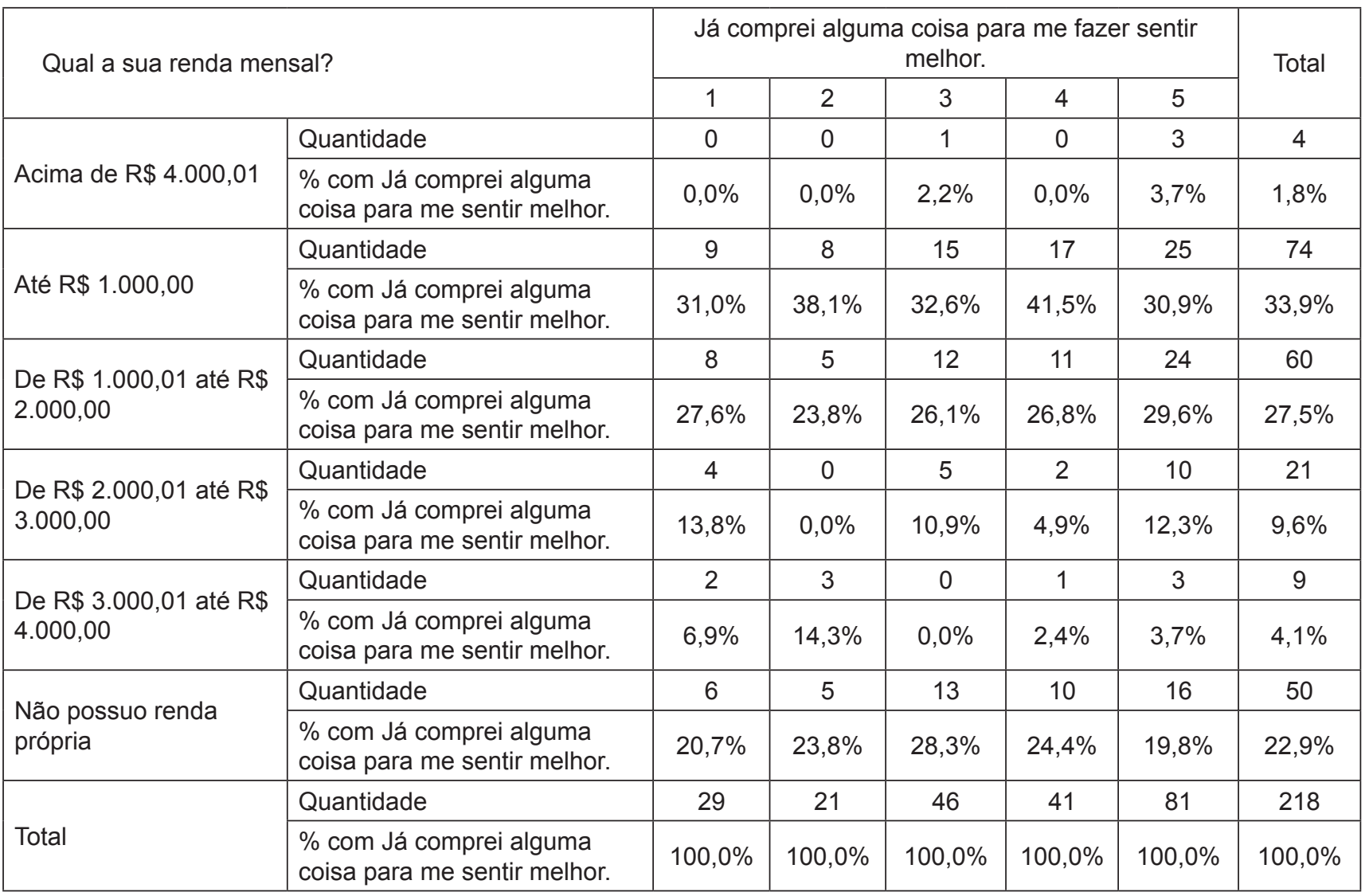

Fonte: Elaborado pelos autores (2016).

\subsection{Análise dos antecedentes das compras compulsivas}

A análise dos dados referentes aos antecedentes das compras compulsivas entre os jovens universitários foi feita utilizando-se a modelagem de equações estruturais, por meio da aplicação de duas abordagens: análise fatorial confirmatória e análise de caminhos. A análise fatorial confirmatória foi utilizada para agrupar as variáveis observadas nas seguintes variáveis latentes: poder/prestígio, ansiedade, desconfiança e compra compulsiva. Por sua vez, a análise de caminhos foi utilizada para analisar a relação entre a variável compra compulsiva e as variáveis poder/prestígio, ansiedade e desconfiança.

Após essa definição inicial, elaborou-se um modelo no software AMOS, a partir do qual foi possível testar a significância e o poder de explicação de cada um dos antecedentes teóricos das compras compulsivas aqui analisados. O modelo estruturado contou com as quatro variáveis latentes já citadas, em que uma delas é endógena (compra compulsiva) e as demais, exógenas, além de vinte variáveis observadas.

Cada uma das variáveis observadas foram relacionadas a um erro, o que se justifica com base no fato de que tais variáveis, que podem ser medidas, não descrevem perfeitamente aquelas que não são mensuradas diretamente, denominadas latentes (SILVA, 2006).

Para avaliar a qualidade do ajuste obtido, que se refere ao grau de correspondência entre a matriz de dados reais (dados de entrada ou observados) e a matriz de dados previstos (HAIR JUNIOR et al., 2009), foram utilizadas três medidas, sendo elas de ajuste absoluto, incremental e parcimonioso.

A qualidade do ajuste absoluto, que corresponde ao grau em que o modelo desenvolvido consegue prever a matriz de correlações observada, foi analisada com base na raiz do erro quadrático médio de aproximação (RMSEA ou Root Mean Square Error of Aproximation), que aponta a discrepância por grau de liberdade caso o modelo fosse estimado na população, recomendando-se um valor não superior a 
0,08 (GARSON, 2012; ARBUCKLE, 2010; HAIR JUNIOR et al., 2009). Para o modelo desenvolvido nesta pesquisa, o RMSEA foi de 0,082 (ver quadro 3), portanto, um pouco acima do valor limítrofe, o que o torna, do ponto de vista do ajuste absoluto alcançado, perifericamente aceitável.

Quadro 3 - Índices de qualidade de ajuste absoluto

\begin{tabular}{|l|l|l|l|l|}
\hline Modelo & RMSEA & LO 90 & HI 90 & PCLOSE \\
\hline Modelo padrão & $\mathbf{0 , 0 8 2}$ & 0,072 & 0,092 & 0,000 \\
\hline Modelo independente & 0,167 & 0,158 & 0,175 & 0,000 \\
\hline
\end{tabular}

Fonte: Output do AMOS (2016).

De forma complementar, a qualidade do ajuste incremental, que resulta da comparação entre o modelo desenvolvido e um modelo de referência que apresenta um único constructo sendo medido por todos os indicadores, foi analisada com base no índice de qualidade de ajuste calibrado (AGFI ou adjusted goodness-of-fit index), cujo valor varia entre zero (ajuste pobre) e um (ajuste perfeito) (GARSON, 2012; ARBUCKLE, 2010; HAIR JUNIOR et al., 2009). Para o modelo testado, obteve-se um AGFI de 0,840 (ver quadro 4), sendo considerado aceitável por estar próximo de um.

Quadro 4 - Índices de qualidade de ajuste incremental

\begin{tabular}{|l|c|c|c|c|}
\hline Modelo & RMR & GFI & AGFI & PGFI \\
\hline Modelo padrão & 0,163 & 0,866 & $\mathbf{0 , 8 4 0}$ &, 673 \\
\hline Modelo saturado & 0,000 & 1,000 & & \\
\hline Modelo independente & 0,311 & 0,518 & 0,486 &, 461 \\
\hline
\end{tabular}

Fonte: Output do AMOS (2016).

Por fim, a qualidade do ajuste parcimonioso, que aponta se houve superajustamento dos dados devido ao excesso de coeficientes no modelo testado, foi analisada com base no valor do qui-quadrado normado, que exprime a razão entre o qui-quadrado e o número de graus de liberdade obtidos, sendo recomendado um valor inferior a cinco (SCHUMACKER; LOMAX, 2004; JORESKOG, 1970). Para o modelo desenvolvido, obteve-se o valor de 2,457 (ver quadro 5), o que atende às condições supracitadas e evidencia bom ajuste parcimonioso.

Quadro 5 - Índices de qualidade de ajuste parcimonioso

\begin{tabular}{|l|c|c|c|c|c|}
\hline Modelo & NPAR & CMIN & DF & P & CMIN/DF \\
\hline Modelo padrão & 46 & 402,881 & 164 &, 000 & $\mathbf{2 , 4 5 7}$ \\
\hline Modelo saturado & 210 & 0,000 & 0 & & \\
\hline Modelo independente & 20 & 1337,422 & 190 &, 000 & 7,039 \\
\hline
\end{tabular}

Fonte: Output do AMOS (2016).

Analisando conjuntamente a qualidade dos ajustes absoluto, incremental e parcimonioso, concluise que o modelo proposto é válido, aceitável e capaz de explicar a matriz de dados observados. Sendo assim, seguiu-se para a análise dos coeficientes de regressão obtidos na análise de caminhos.

Primeiramente, constatou-se que todas as variáveis latentes apresentaram relação positiva e estatisticamente significativa com as variáveis observadas que lhes deram origem (ver quadro 6). Com isso, verifica-se que as variáveis de pesquisa conseguiram explicar de forma satisfatória as variáveis latentes do estudo (compra compulsiva, poder/prestígio, ansiedade e desconfiança). 
Quadro 6 - Relações testadas no modelo de equações estruturais

\begin{tabular}{|cccccccc}
\hline & & & & & \\
& & & Estimativa & Erro padrão & Valor crítico & P \\
\hline $\begin{array}{c}\text { Compra__ } \\
\text { compulsiva }\end{array}$ & $\leftarrow$ & Ansiedade & 0,727 & 0,318 & 2,285 & 0,022 \\
Compra__ & $\leftarrow$ & Desconfiança & $-0,232$ & 0,083 & $-2,784$ & 0,005 \\
compulsiva & $\leftarrow$ & & & & \\
Compra_ & $\leftarrow$ & Poder_prestígio & $-0,039$ & 0,035 & $-1,107$ & 0,268 \\
compulsiva & $\leftarrow$ & Compra_compulsiva & 1,000 & & & \\
CC6 & $\leftarrow$ & Compra_compulsiva & 2,378 & 0,603 & 3,941 & 0,000 \\
CC5 & $\leftarrow$ & Compra_compulsiva & 1,849 & 0,564 & 3,281 & 0,001 \\
CC4 & $\leftarrow$ & Compra_compulsiva & 1,457 & 0,410 & 3,557 & 0,000 \\
CC3 & $\leftarrow$ & Compra_compulsiva & 2,677 & 0,683 & 3,920 & 0,000 \\
CC2 & $\leftarrow$ & Compra_compulsiva & 2,647 & 0,684 & 3,867 & 0,000 \\
CC1 & $\leftarrow$ & Poder_prestígio & 1,000 & & & \\
PP1 & $\leftarrow$ & Poder_prestígio & 0,514 & 0,067 & 7,671 & 0,000 \\
PP2 & $\leftarrow$ & Poder_prestígio & 0,969 & 0,084 & 11,575 & 0,000 \\
PP3 & $\leftarrow$ & Poder_prestígio & 0,937 & 0,093 & 10,028 & 0,000 \\
PP4 & $\leftarrow$ & Poder_prestígio & 0,990 & 0,107 & 9,237 & 0,000 \\
PP5 & $\leftarrow$ & Ansiedade & 1,000 & & & \\
A4 & $\leftarrow$ & Ansiedade & 2,915 & 1,024 & 2,847 & 0,004 \\
A3 & $\leftarrow$ & Ansiedade & 2,107 & 0,788 & 2,674 & 0,007 \\
A2 & $\leftarrow$ & Ansiedade & 2,627 & 0,945 & 2,780 & 0,005 \\
A1 & $\leftarrow$ & Desconfiança & 1,000 & & & \\
D5 & $\leftarrow$ & Desconfiança & 1,270 & 0,304 & 4,184 & 0,000 \\
D4 & $\leftarrow$ & Desconfiança & 1,484 & 0,333 & 4,462 & 0,000 \\
D3 & $\leftarrow$ & Desconfiança & 0,945 & 0,270 & 3,493 & 0,000 \\
D2 & $\leftarrow$ & Desconfiança & 1,210 & 0,297 & 4,067 & 0,000 \\
D1 & $\leftarrow$ & & & & \\
\hline
\end{tabular}

Fonte: Output do AMOS (2016).

Posteriormente, ao se analisar o p-valor das relações entre as variáveis latentes exógenas (poder/ prestígio, ansiedade e desconfiança) e a variável latente endógena (compra compulsiva) (ver quadro 6), concluiu-se que apenas a variável poder/prestígio não possuía relação estatisticamente significativa com a variável compra compulsiva ( $p$-valor de 0,268 ) e, por isso, o coeficiente de regressão obtido não foi analisado.

A relação entre a variável ansiedade e a variável compra compulsiva apresentou p-valor de 0,022 e coeficiente de regressão padronizado de 0,727 . Já a relação entre a variável desconfiança e a variável compra compulsiva apresentou p-valor de 0,005 e coeficiente de regressão padronizado de $-0,232$, conforme se verifica no quadro 6 e na figura 2 a seguir 
Figura 2- Modelo de equações estruturais proposto

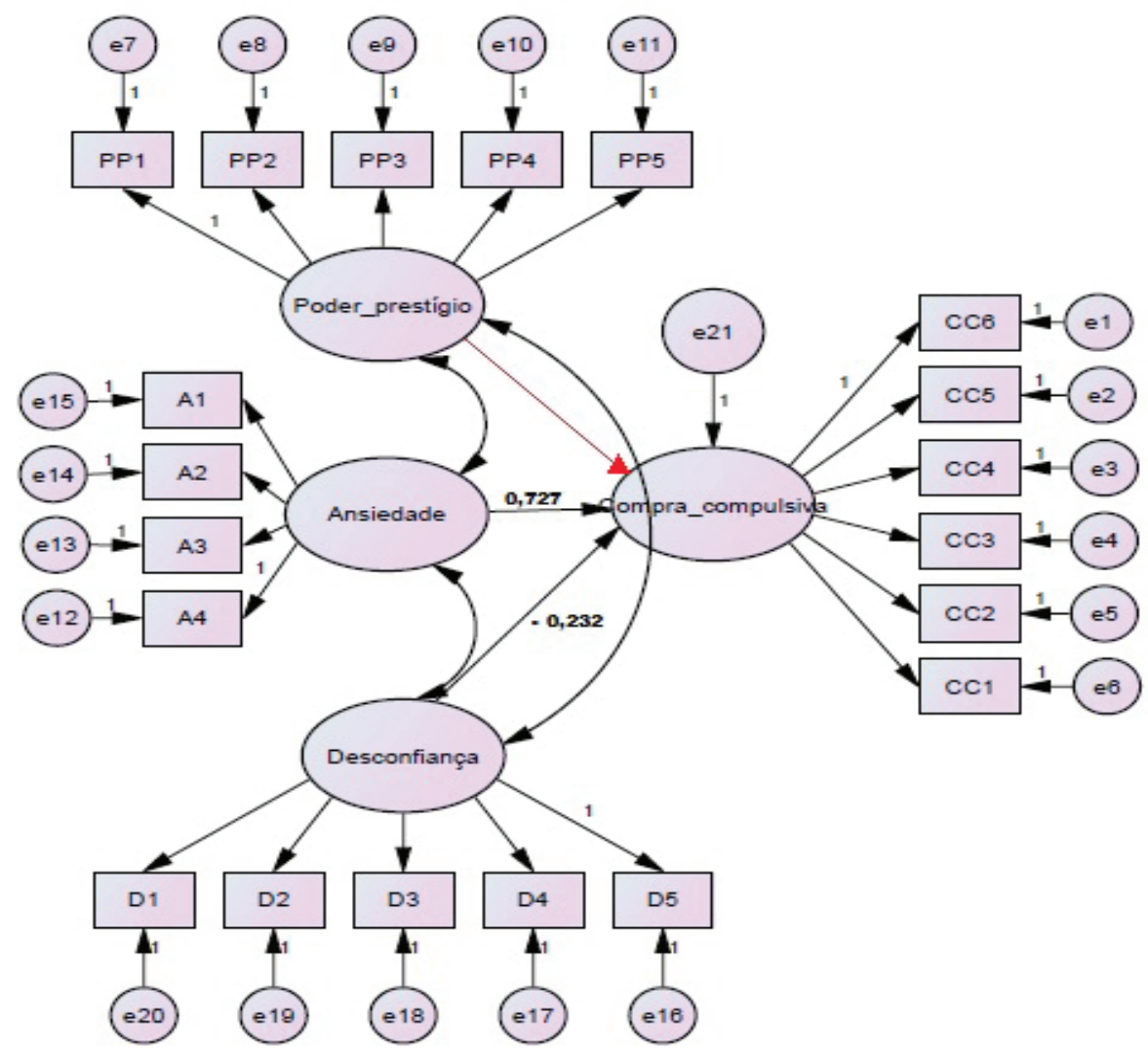

Fonte: Elaborado pelos autores (2016).

Analisando-se os coeficientes obtidos, pode-se concluir que, entre os jovens universitários que fizeram parte da amostra, a ansiedade exerce influência positiva sobre o comportamento de comprar compulsivamente, enquanto a desconfiança exerce influência negativa. Portanto, universitários mais ansiosos e menos desconfiados tendem a comprar mais de forma compulsiva.

Tal constatação corrobora os achados de Stefanini e Oliveira (2014), Kukar-Kinner, Ridgway e Monroet (2012) e Yamauchi e Templer (1982), que apontam que indivíduos mais desconfiados, reticentes em relação a sua própria capacidade de tomar decisões de consumo, aos preços praticados e possíveis vantagens que irão auferir ao adquirirem determinado produto, tendem a ser menos compulsivos no momento de comprar.

Além disso, os resultados aqui obtidos vêm ao encontro daqueles alcançados por Verplanken et al. (2005), Roberts e Jones (2001), Edwards (1993) e Yamauchi e Templer (1982), que apontam que indivíduos que apresentam elevado nível de ansiedade veem no consumo compulsivo uma válvula de escape e, consequentemente, mostram-se mais propensos a comprar sem que haja necessidade. Acrescenta-se que os resultados obtidos por Roberts e Roberts (2012) reforçam os resultados aqui encontrados e discutidos, pois esses autores relatam que os adolescentes compram compulsivamente para lidar com os altos níveis de estresse acadêmico, sendo uma estratégia de enfrentamento comum a ambos os sexos.

Finalizando, destaca-se que a relação entre a busca por poder e prestígio e o comportamento de compra compulsiva não foi validada estatisticamente neste estudo, não sendo possível corroborar os resultados de Roberts e Roberts (2012), Oliveira, Ikeda e Santos(2004) e Roberts e Jones (2001), que indicam que os indivíduos, com vistas a alcançarem maior prestígio perante a sociedade e grupos aos quais pertencem, tendem a comprar para simbolizar seu poder financeiro, e não somente para suprir suas necessidades. Nesse sentido, recomenda-se que essa relação seja investigada com maior nível de profundidade em outras pesquisas cujo público-alvo seja os universitários brasileiros. 


\section{Conclusão}

O consumo não se resume mais ao ato de consumir e dispor de produtos e serviços. Nos dias atuais, tal atividade extrapola a função utilitarista e passa a ter a função de criar, moldar e externalizar a identidade dos indivíduos, cada vez mais preocupados com sua imagem perante a sociedade, assim como de gerar prazer e atenuar desconfortos de ordem psicológica.

Nesse sentido, este estudo teve como objetivo investigar os antecedentes do comportamento compulsivo de compra entre o público universitário de uma instituição federal de ensino localizada em Mariana (MG). Dentre os vários fatores que levam os indivíduos a comprarem compulsivamente, foram utilizados três como base para esta pesquisa: busca por poder e prestígio, ansiedade e desconfiança.

A partir da revisão de literatura realizada e da análise dos resultados obtidos, foi possível confirmar a ansiedade e a desconfiança como antecedentes do comportamento de compra compulsiva entre os jovens universitários, sendo que o primeiro exerce influência positiva, e o segundo, negativa.

Sendo assim, conclui-se que os universitários mais ansiosos, que se mantêm aflitos e agoniados por períodos prolongados, tendem a lidar com esse desconforto psíquico dedicando-se ao consumo desregrado e desvinculado de suas necessidades.

Por outro lado, universitários mais desconfiados em relação às ofertas que são apresentadas pelas empresas, sensíveis aos preços praticados por elas e ainda cientes de suas limitações enquanto tomadores da decisão de consumo tendem a se mostrar mais reticentes no momento da compra e, consequentemente, a não comprar compulsivamente.

Tais conclusões evidenciam que, para reverter a tendência de crescimento no número de indivíduos endividados no Brasil, que se observa na atualidade, é preciso habituar as famílias e instituições de ensino a despenderem tempo e atenção à educação financeira das crianças e jovens. Com isso, espera-se maior consciência e desconfiança no momento de comprar, o que poderia reduzir a compulsão consumista durante fases posteriores da vida.

Igualmente importante se faz a reflexão acerca dos motivos que levam os indivíduos a experimentarem tão elevada ansiedade desde muito cedo, que se torna um distúrbio psicológico e tende a gerar um desequilíbrio grave não só no âmbito financeiro da vida das pessoas, mas também nos âmbitos social e profissional.

Por fim, faz-se necessário que os paradigmas vigentes na sociedade moderna sejam profundamente questionados com o intuito de se discutir a importância que atribuída aos bens materiais, a qual gera angústia e ansiedade crescentes nos jovens brasileiros, que se veem pressionados a ingressarem prematuramente na corrida do "ter" ao invés do "ser".

Para estudos futuros, sugere-se que seja analisada a relação das compras compulsivas com o uso do cartão de crédito, de forma que seja investigado até que ponto ele é um facilitador para o surgimento/ desenvolvimento do comportamento de consumo vicioso.

\section{Referências}

ABECS. Brasileiro gasta em média R\$ 78 por compra no cartão de crédito em 2015. Disponível em: <http://www.abecs.org.br/abecs-noticias/mercado-de-cartoes>. Acesso em: 02 Set.2015.

ARBUCKLE, J. L. IBM SPSS AMOS 19 user's guide. Chicago: IBM, 2010. Disponivel em: <https:// pt.scribd.com/doc/49697749/IBM-SPSS-Amos-19-User-s-Guide>. Acesso em: 02 Set.2015.

BAKER, A.; MATHUR, A.; FATT, C. K.; MOSCHIS, G. P.; RIGDON, E. E. Using the life course paradigm to explain mechanisms that link family disruptions to compulsive buying. Journal of Consumer Affairs, [S.I], v. 47, n. 2, p. 263-288, 2013.

BYRNE, D. G.; DAVENPORT, S. C.; MAZANOV, J. Profiles of adolescent stress: the development of the adolescent stress questionnaire (ASQ). Journal of Adolescence, [S.I], v. 30, n. 3, p. 393-416, 2007. 
BEUTLER, I. F.; GUDMUNSON, C. G. New adolescent Money Attitude Scale: entitlement and conscientiousness. Journal of Financial Counseling and Planning, [S.I], v. 23, n. 2, p. 18-31, 2012.

BLACKWELL, R. D.; MINIARD, P. W.; ENGEL, J. F. Comportamento do consumidor. 9. ed. São Paulo: Pioneira Thomson Learning, 2005.

DE MEDEIROS, F. G.; DINIZ, I. S. F. N.; Da COSTA, F. J.; PEREIRA, R. D. C. F.Influência de estresse, materialismo e autoestima na compra compulsiva de adolescentes. Revista de Administração Contemporânea, [S.I], v. 19, p. 137, 2015.

DITTMAR, H.; LONG, K.; BOND, R. When a better self is only a button click away: associations between materialistic values, emotional and identity-related buying motives, and compulsive buying tendency online. Journal of Social and Clinical Psychology, [S.I], v. 26,n. 3, p. 334-361, 2007.

EDWARDS, E. A. Development of a new scale for measuring compulsive buying behavior. Financial Counseling and Planning, [S.I], v. 4, p. 67-85, 1993.

ELLIOTT, R. Addictive consumption: function and fragmentations in post modernity. Journal of Consumer Policy, [S.I], v. 17, n.2, p. 159-179, 1994.

GARSON, G. D. Structural Equation Modeling. Asheboro: Statistical Publishing Associates, 2012. HAIR JUNIOR, J. F.; BABIN, B.; MONEY, A. R.; SAMOUEL, P. Fundamentos de métodos de pesquisa em administração. Porto Alegre: Bookman, 2005.

HAIR JUNIOR, J. F.; BLACK, W. C.; BABIN, B. J.; ANDERSON, R. E.; TATHAM, R. L. Análise multivariada de dados. 6. ed. Porto Alegre: Bookman, 2009.

HANNA, N.; WOZNIAK, R.; HANNA, M. Consumer behavior: an applied approach. 3.ed. Dubuque: Kendall Hunt Publishing Company, 2009.

HUTCHINSON, S. L.; BALDWIN, C. K.; OH, S.-S. Adolescent coping: exploring adolescents' leisurebased responses to stress. Leisure Sciences, [S.I], v. 28, n. 2, p. 115-131, 2006.

KUNKEL, F. I. R.; VIEIRA, K. M.; POTRICH, A. C. G. Causas e consequências da dívida no cartão de crédito: uma análise multifatores. Revista de Administração, São Paulo, v. 50, n.2, p.169-182, 2015.

KUKAR-KINNEY, M.; RIDGWAY, N. M.; MONROE, K. B. The role of price in the behavior and purchase decisions of compulsive buyers. Journal of Retailing, [S.I], v. 88, n. 1, p. 63-71, 2012.

JORESKOG, K. G. A general method of analysis of covariance structures. Biometrik, [S.I], v. 57, n. 2, p. 239-251, 1970.

LEITE, P. L.; RANGÉ, B. P.; JUNIOR, R. D. C. R.; FERNANDEZ, J. L.; CARDOSO, A.; SILVA, O. E. Validação e aferição de fidedignidade da versão brasileira da Compulsive Buying Scale. Revista de Psiquiatria Clínica, [S.I], v. 39, n. 3, p. 100-105, 2012.

MALHOTRA, N. K. Pesquisa de marketing: uma orientação aplicada. Porto Alegre: Bookman, 2006.

MANOLIS, C.; ROBERTS, J. A. Subjective well-being among adolescent consumers: the effects of materialism, compulsive buying, and time affluence. Applied Research in Quality of Life, [S.I], v. 7, n. 2, p. 117-135, 2012.

MEDEIROS, F. G.; DINIZ, I. S. F. N.; COSTA, F. J.; PEREIRA, R. C. F. Influência de estresse, materialismo e autoestima na compra compulsiva de adolescentes. RAC-Eletrônica, Curitiba, v. 19, n. 2, p. 137-156, Aug. 2015.

MERLO, E. M.; CERIBELI, H. B. Comportamento do consumidor. Rio de Janeiro: LTC, 2014.

MITTAL, B.; E HOLBROOK, M. ;BEATTY, S.; RAGHUBIR, P.; WOODSIDE, A. G.Consumer behavior: how humans think, feel, and act in the marketplace. Cincinnati: Open Mentis, 2008.

MOWEN, J. C.; MINOR, M. S. Comportamento do consumidor. São Paulo: Pearson Prentice Hall, 2003. 
OLIVEIRA, T. M. V.; IKEDA, A. A.; SANTOS, R. C. Compra compulsiva e a influência do cartão de crédito. RAE, [S.I], v. 44, n. 3, p. 89-99, jul./set. 2004.

PALAN, K. M.; MORROW, P. C.; TRAPP, A.; BLACKBURN, V. Compulsive buying behavior in college students: the mediating role of credit card misuse. The Journal of Marketing Theory and Practice, [S.I], $v$. 19, n. 1, p. 81-96, 2011.

ROBERTS, J. A.; JONES, E. Money attitudes, credit card use, and compulsive buying among American college students. The Journal of Consumer Affairs, [S.I], v. 35, n. 2, p. 213-240, 2001.

ROBERTS, J. A.; ROBERTS, C. Stress, gender and compulsive buying among early adolescents. Young Consumers: Insight and Ideas for Responsible Marketers, [S.I], v. 13, n. 2, p. 113-123, 2012.

ROBERTS, J. A. Compulsive buying among college students: an investigation of its antecedents, consequences, and implications for public policy. Journal of Consumer Affairs, [S.I], v. 32, n.2, p. 295308, 1998.

ROOK, D. W.; FISHER, R. J. Normative influences on impulsive buying behavior. The Journal of Consumer Research, [S.I], v. 22, n. 3, p. 305-313, 1995.

SCHUMACKER, R. E.; LOMAX, R. G. A beginner's guide to structural equation modeling. 2. ed. Mahwah: Lawrence Erlbaum Associates, 2004.

SHETH, J. N.; MITTAL, B.; NEWMAN, B. I. Comportamento do consumidor: indo além do comportamento do consumidor. São Paulo: Atlas, 2001.

SILVA, J. S. F. Modelagem de Equações Estruturais: apresentação de uma metodologia. Porto Alegre: UFRGS, 2006. 105f. Dissertação (Mestrado) - Programa de Pós-Graduação em Engenharia de Produção, Universidade Federal do Rio Grande do Sul, Porto Alegre, 2006.

SOLOMON, M. R. O comportamento do consumidor: comprando, possuindo e sendo. 7. ed. Porto Alegre: Bookman, 2008.

STEFANINI, C. J.; OLIVEIRA, B. Fatores relacionados à compra compulsiva. In: EnANPAD, 38., 2014, Rio de Janeiro. Anais... Rio de Janeiro: ANPAD, p. 1-14, 2014.

VALENCE, G.; D'ASTOUS, A.; FORTIER, L. Compulsive buying: concept and measurement. Journal of Consumer Policy, [S.I],v. 11, n. 4, p. 419-433, 1998.

VERPLANKEN, B.; HERABADI, A. G.; PERRY, J. A.; SILVERA, D. H. Consumer style e health: the role of impulsive buying in unhealthy eating. Psychology e Health, [S.I], v. 20, n. 4, p. 429-441, 2005.

VERPLANKEN, B.; SATO, A. The psychology of impulse buying: an integrative self-regulation approach. Journal of Consumer Policy, [S.I], v. 34, n. 2, p. 197-210, 2011.

VIEIRA, K. M.; PARABONI, A. L.; CAMPARA, J. P.; POTRICH, A. C. G.; KUNKEL, F. I. R. O uso do cartão de crédito por universitários: análise do perfil, da compra compulsiva e do conhecimento financeiro. Estudo \& Debate, Lajeado, v. 21, n. 2, p. 100-122, 2014.

WAGNER, J.; LÜDTKE, O.; JONKMANN, K.; TRAUTWEIN, U. Cherish yourself: longitudinal patterns and conditions of self-esteem change in the transition to young adulthood. Journal of Personality and Social Psychology, [S.I], v. 104, n. 1, p.148-163, 2013.

WEINBERG, P.; GOTTWALD, W. Impulsive consumer buying as a result of emotions. Journal of Business Research, [S.I],v. 10, n. 1, p. 43-57, 1982.

YAMAUCHI, K.; D. TEMPLER. The development of aMoney Attitudes Scale. Journal of Personality Assessment, [S.I], v. 46, n. 5, p. 522-528, 1982.

Data de Submissão: $17 / 5 / 16$

Data de Aprovação: 9/4/17 